A N T O N I O TR A M P U S

\title{
FILANGIERI IN AUSTRIA: LA TRADUZIONE PERDUTA DELLA "SCIENZA DELLA LEGISLAZIONE"
}

Da qualche anno la storiografia sull'Illuminismo in Italia sta conoscendo un significativo mutamento di tendenza, rispetto ad una tradizione che vedeva un notevole impegno nella stesura e nella pubblicazione di biografie e saggi critici, ma un sostanziale disimpegno rispetto alla conoscenza dei testi e alle edizioni di fonti. Questa nuova stagione, inaugurata con la pubblicazione degli "Scritti economici" di Antonio Genovesi, per cura di Maria Luisa Perna ${ }^{1}$, è giunta ora ad interessare i tre principali protagonisti della stagione dei lumi nella penisola italiana, ora al centro di significative iniziative editoriali. Si tratta di Cesare Beccaria, la cui edizione nazionale delle "Opere", avviata nel 1984 con gli auspici di Mediobanca, è tutt'oggi in $\operatorname{corso}^{2}$; di Pietro Verri, la cui edizione nazionale degli scritti, promossa nel 1997 dalla Fondazione Raffaele Mattioli, si è concretizzata ora nella pubblicazione di un primo volume ${ }^{3 ;}$ di Gaetano Filangieri, la cui "Scienza della legislazione", in edizione critica, ha finalmente raggiunto fra il 2003 e il 2004 la pubblicazione di tutti i volumi, grazie al concorso del Centro di studi sull'Illuminismo europeo e della Compagnia di San Paolo ${ }^{4}$.

1 A. Genovesi, Scritti economici, a cura di M. L. Perna. 2 voll., Napoli 1984; ma è di imminente pubblicazione anche l'edizione delle Lezioni di commercio, sempre a cura di M. L. Perna. Napoli 2004.

2 Edizione nazionale delle opere di Cesare Beccaria, diretta da L. Firpo e G. FranCIONI. Milano 1984. Il primo volume contiene l'edizione di "Dei delitti e delle pene"; l'ultimo publicato, l'ottavo (Milano 1993) contiene gli "Atti di governo" (serie III, 1784-1786), a cura di R. CANETTA.

3 Edizione nazionale delle opere di Pietro Verri, Roma, Edizioni di Storia e letteratura, 2003; il primo volume pubblicato, che è in realtà il quinto della serie, contiene gli "Scritti di argomento familiare e autobiografico", a cura di G. BARBARISI.

${ }^{4}$ G. Filangieri, La scienza della legislazione. Edizione critica, diretta da V. FerRoNe (Centro di Studi sull'Tlluminismo europeo. Studi e testi 1). Venezia 2003-2004, apparsa in sette volumi. 
La possibilità di rimettere al centro della discussione nazionale e internazionale i testi dei maggiori illuministi italiani, non casualmente legata all'impegno finanziario di grandi fondazioni bancarie e a quello scientifico di comitati di sicuro prestigio, ha innescato quindi un nuovo circolo virtuoso che permette di riaprire il dibattito tornando a lavorare direttamente sui testi, disponibili in affidabili edizioni commentate. In questo modo, appare maggiormente verificabile la validità di un percorso culturale delineato già quarant'anni or sono da Franco Venturi ${ }^{5}$, che inseriva la cultura italiana del Settecento in un più ampio contesto europeo ed internazionale, e che è venuto arricchendosi nel tempo con significativi approfondimenti e contributi critici che hanno concorso a rivisitare - attraverso inedite prospettive metodologiche e critiche - personalità altrimenti già note al dibattito scientifico ${ }^{6}$. Queste iniziative assumono, com'è evidente, un'importanza notevole per le ricadute sullo spazio specificamente italiano, ma pongono allo stesso tempo un nuovo e altrettanto affascinante problema, che è quello di poter apprezzare il contributo italiano alla dimensione europea dei lumi, nella misura in cui le opere di questi autori circolarono e vennero tradotte al di fuori della penisola, influenzando o meno la cultura dei paesi nei quali vennero lette e discusse.

Da questo punto di vista, l'antico progetto di studio profilato da Venturi sulla "circolazione" delle idee si carica di valenze nuove nel momento in cui viene radicato all'interno del contesto che ormai viene delineandosi: quello nel quale la discussione non riguardava più soltanto le spinte riformatrici ma involgeva ormai il problema di un diverso significato politico del repubblicanesimo, della nascita del costituzionalismo e delle teorie dei diritti dell'uomo. Lo studio della circolazione e della fortuna internazionale dell'opera di Gaetano Filangieri (1753-1788), che si è accompagnato all'edizione critica della "Scienza della legislazione" , si

\footnotetext{
5 A partire dal primo volume dell'opera "Illuministi italiani". Riformatori lombardi, piemontesi e toscani, a cura di F. Venturi (La letteratura italiana. Storia e testi 46). Milano-Napoli 1958, e in quelli apparsi poi negli anni successivi; si veda però anche F. Venturi, L'Italia fuori d'Italia, in: Storia d'Italia. Annali 3. Dal primo Settecento all'Unità. Torino 1973, 1069-1119.

${ }^{6}$ Segnalo a titolo di esempio e di confronto C. CAPRA, I progressi della ragione. Vita di Pietro Verri (Collezione di Testi e di Studi. Storiografia). Bologna 2003 e V. Ferrone, La società giusta ed equa. Repubblicanesimo e diritti dell'uomo in Gaetano Filangieri (Collezione storica Laterza). Roma-Bari 2003.

${ }^{7}$ L'attenzione per l'opera di Filangieri si inserisce nel quadro più ampio di un progetto nato a partire dall'edizione critica della Scienza della legislazione, promossa dal Centro di Studi sull'Tlluminismo europeo di Venezia, con il concorso della Compagnia di San Paolo e del Ministero per l'Istruzione, Università e
} 
colloca proprio all'interno di questo quadro e può recare un contributo originale all'approfondimento del problema storiografico dell'Illuminismo italiano nel contesto europeo.

La "Scienza della legislazione", composta di cinque parti suddivise in sette volumi, venne stesa negli anni Ottanta del Settecento (1780-1787), in una fase cruciale per la cultura occidentale, segnata dall'irrompere della politica e dalla trasformazione delle utopie settecentesche in nuovi progetti di governo, sull'onda della Rivoluzione americana (1776). Del testo di Filangieri risultano censite sino ad oggi 73 edizioni e traduzioni, una trentina delle quali risalenti al periodo fra 1780 e il 1814, che si prestano assai bene a questo tipo di indagine, anche in ragione dei temi affrontati dall'illuminista napoletano. Attraverso il complicato meccanismo e intreccio delle riedizioni e delle traduzioni in tutti i paesi europei, l'opera rappresenta un esempio importante della notevole circolazione internazionale, sul finire del Settecento e all'inizio dell'Ottocento, di idee e di valori maturati attraverso il confronto internazionale, ma per larga misura tributari anche della tradizione giuridica e politica italiana.

Le ragioni di questa fortuna sono molteplici e derivano da molti fattori, tra cui spiccano i contenuti, la tecnica, il linguaggio dell'opera di Filangieri. Le cinque parti della "Scienza della legislazione" affrontavano le questioni centrali nel dibattito politico del tardo Settecento e del primo Ottocento: la definizione del concetto di "scienza legislativa" e di "legge generale ed astratta", opposta al particolarismo dell'Antico regime; il sistema dei rapporti politici all'interno dello Stato; la natura delle leggi economiche e la loro funzione all'interno di un mercato in espansione; la riforma del processo penale e la qualificazione dei delitti e delle pene; il miglioramento dell'educazione e la nascita di un'istruzione pubblica e generalizzata; il rapporto dell'individuo con la religione e della religione con lo Stato. Si tratta di una straordinaria sintesi dell'intera tradizione dell'Illuminismo europeo, che però, dal punto di vista contenutistico, non rimaneva a livello di una filosofia della politica o della storia, ma diveniva un vero e proprio progetto giuridico per l'individuazione dei princìpi che di lì a poco sarebbero stati poi formulati e accolti nelle carte costituzionali dell'ultimo Settecento e del primo Ottocento.

Infine, acquistava molta importanza anche il linguaggio utilizzato dall'illuminista napoletano. Per raggiungere questi obiettivi, per misurarsi con coloro che l'avevano preceduto, l'autore della "Scienza della legislazione" si trovò costretto ad operare attraverso il linguaggio politi-

Ricerca (MIUR) e ora entrato in una seconda fase, dedicata allo studio del linguaggio dei diritti fra Antico Regime ed età della Restaurazione. 
co del suo tempo, verificandone l'utilità e l'adeguatezza rispetto alle idee che intendeva esprimere. Introdusse e definì però nuovi significati a partire da un lessico antico, per adattarlo ad una prosa asciutta ed essenziale che servisse a rendere tali concetti immediatamente comprensibili ad un'opinione pubblica sempre più attenta ed informata. Sul piano linguistico, così, la "Scienza della legislazione" veniva a rappresentare un importante tentativo di mettere a fuoco aspetti significativi del linguaggio dei lumi e della sua rielaborazione negli anni Ottanta, legati alla nascita di nuove parole e di nuove espressioni (tra cui ad esempio quelle di "opinione pubblica", di “costituzione", di "diritti dell'uomo"), oppure all'adattamento di parole più antiche a nuovi significati (tipica è la trasformazione della parola "Illuminismo" dal classico significato di "illuminazione per grazia divina" a "illuminazione per mezzo della ragione").

Appare straordinario allora il fatto che un'opera così complessa sul piano concettuale e così articolata su quello editoriale (cinque libri, come si è detto, a differenza del più sintetico volumetto scritto da Becaria vent'anni prima), non solo ottenesse un grande successo misurabile attraverso le riedizioni e le ristampe italiane, ma vedesse impegnati sul fronte internazionale un ampio numero di traduttori e di editori stranieri. La possibilità di studiare un testo come "La scienza della legislazione", elaborato nella fase più intensa del dibattito politico tardo illuministico, e di poter disporre delle sue traduzioni coeve in tedesco, inglese, francese, spagnolo, svedese, russo, rappresenta perciò un'occasione importante per conoscere meglio - accanto al testo originario - le declinazioni del movimento dei lumi nel continente europeo.

Gli spunti per questo tipo di ricerca nascono inoltre anche dall'incontro - di cui solo in tempi relativamente recenti si è presa consapevolezza - fra gli strumenti metodologici propri della storia della cultura e quelli offerti dalla linguistica storica. Oggi è possibile indagare e comprendere meglio i meccanismi di diffusione e di circolazione dei testi, di traduzione e adattamento ai contesti culturali specifici dei diversi paesi europei, le strategie editoriali a questi sottesi, le scelte dei traduttori e le trasformazioni dei significati lessicali e linguistici. La linguistica storica aveva già da tempo segnalato queste ipotesi di lavoro ${ }^{8}$, attraverso un confronto più diretto fra storici e linguisti, tuttavia il percorso rimaneva in gran parte nuovo e poco esplorato dal punto di vista storico. Alcuni contributi, apparsi in tempi anche recenti, hanno fatto intravvedere le prospettive

${ }^{8}$ R. Robin, Histoire et linguistique (Linguistique). Paris 1973; A. Grafton, The Footnote. A Curious History. Harvard 1997. 
offerte dallo studio dei processi di mediazione linguistica nell'età moderna ${ }^{9}$, ma anche i rischi, evidenti dalla prospettiva del lavoro storiografico, di ridurre questo tipo di ricerca ad un'analisi delle parole avulsa da un più ampio contesto culturale e politico nel quale vennero elaborate ${ }^{10}$.

Bisogna anche rilevare, però, che solo in tempi molto recenti si è assistito ai primi tentativi di trasferire queste ipotesi interpretative sul piano della ricerca storica, andando a verificare attraverso indagini mirate i mutamenti complessivi del linguaggio e del vocabolario settecentesco. È vero che sinora all'interno di quest'ambito si è comunque privilegiata la ricerca sul vocabolario politico rispetto a quella sul vocabolario giuridico vero e proprio. La storiografia italiana si è concentrata piuttosto su questo primo aspetto, con alcune incursioni circoscritte nello spazio cronologico per focalizzare le trasformazioni lessicali nei pochi anni dell'esperienza repubblicana ${ }^{11}$. Nell'ambito austriaco, tuttavia, la situazione non può dirsi certo migliore e ci consegna un tema che attende ancora di essere sistematicamente indagato.

D'altra parte, il problema è stato riproposto in tutta la sua importanza assai recentemente proprio dalla storiografia tedesca. Ripartendo dall'esperienza inaugurata dai "Geschichtliche Grundbegriffe", si è svolto nel 1998 ad Heidelberg un convegno su lingua e diritto nell'Illuminismo tedesco (Recht und Sprache in der deutschen Aufklärung) che ha portato alla pubblicazione di un primo volume che affronta tali complesse questioni su un periodo molto più lungo rispetto al passato, a partire dalla "Fachsprache" di Leibniz per giungere allo "Staatsrecht" di Wieland, tenendo presente un percorso segnato dalla pubblicazione di opere fondamentali per l'epoca, dall'"Historisch-Politisch-Juristisches Lexicon" di Johann Christoph Nehring (1694) allo "Juristisches Hand-Buch" di Georg Stephan Wiesand (1762).

${ }^{9}$ P. Burke, Lingua, società e storia (Quadrante Laterza 37). Roma-Bari 1990; S. Blakemore, Burke and the Fall of Language. The French Revolution as Linguistic Event. Hannover-London 1988; I linguaggi politici delle rivoluzioni in Europa XVII-XIX secolo, a cura di E. PII. Firenze 1992.

${ }^{10} \mathrm{Si}$ veda a tale proposito R. J. W. Evans, Il linguaggio della storia e la storia del linguaggio, in: I linguaggi e la storia, a cura di A. Trampus-U. Kindu (Percorsi). Bologna 2003, 11-54, ma più in generale anche gli altri saggi raccolti nel volume. Da considerare anche le prospettive offerte da: Changements politiques et statut des langues. Histoire et épistémologie 1780-1945, a cura di M.-C. KoK EscalLe, F. Melka (Faux titre 206). Amsterdam-Atlanta 2001.

11 E. Leso, Lingua e rivoluzione. Ricerche sul vocabolario politico italiano del triennio rivoluzionario 1796-1799. Venezia 1991; L. Guerci, Istruire nelle verità repubblicane. La letteratura politica per il popolo nell'Italia in rivoluzione (17961799) (Saggi). Bologna 1999. 
Ed è proprio nel quadro di tale progetto che, analizzando lo stile e il testo giuridico del tardo Settecento, Michael Wieczorrek ha segnalato come nel corso degli anni ottanta il problema che si pose ai giuristi di lingua tedesca non fu più solo quello di affrancare definitivamente il loro linguaggio giuridico dal latino e dai calchi francesi, ma quello di individuare un modo di scrivere, una "Schreibart" che riconducesse l'autore e l'opera ad un preciso contesto istituzionale e culturale rimarcandone altresì lo status e la funzione ${ }^{12}$.

Se vale per le opere in lingua tedesca e per il linguaggio adoperato dai giuristi tedeschi, tutto ciò diventa allora ancor più determinante nel momento in cui si passa ad esaminare la storia delle traduzioni e si analizzano i processi traduttivi e i modi con cui vennero trasferiti i significati nella diffusione in area tedesca di testi apparsi originariamente in altre lingue. L'esempio della "Scienza della legislazione" rappresenta così un laboratorio di particolare interesse per addentrarsi in questo mondo.

Non a caso, la storia delle sue riedizioni e traduzioni si identifica in larga parte con la storia del costituzionalismo europeo. Dopo una prima serie di edizioni e traduzioni all'inizio degli ani Ottanta, infatti, la "Scienza della legislazione" ebbe nuove ristampe e versioni in lingue straniere negli anni delle cosiddette rivoluzioni democratiche, tra il 1796 e il 1799: venne ristampata a Venezia (1796), Genova (1798), Roma (1799), Parigi (1796-1799), Copenhagen (1799) e Livorno (1799); solo tre anni più tardi alle traduzioni tedesche, francesi, inglesi, spagnole e danesi, già esistenti, si aggiunse quella russa (1803) e poi quella svedese ${ }^{13}$. Trascorso poi il periodo napoleonico, la "Scienza della legislazione" nuovamente venne stampata e pubblicata negli anni della Restaurazione, nel quadro della nascita del liberalismo europeo e dei primi movimenti costituzionali (Livorno 1812, Madrid 1813, Stoccolma 1814, Palermo 1815, Milano 1817, Firenze 1820, Venezia 1822, Parigi 1822).

Nelle regioni europee di lingua tedesca, il successo dell'opera di Filangieri non fu meno importante, anche se la vicenda rimane ancora in gran parte da ricostruire. Una traduzione apparve ad Ansbach nel 1784,

12 M. Wieczorrek, Stil und Status. Juristisches Schreiben im 18. Jahrhundert, in: Recht und Sprache in der deutschen Aufklärung, a cura di U. Kronauer, J. Garber (Hallesche Beiträge zur Europäischen Aufklärung). Tübingen 2001, 99-112, in particolare 99-100.

${ }^{13}$ Il quadro sino ad oggi più esauriente sulla diffusione della "Scienza della legislazione" è quello offerto nel saggio su "La genesi e le edizioni della "Scienza della legislazione", in: G. Filangieri, La scienza della legislazione. Edizione critica VII. Venezia 2004, 5ss. 
una seconda quello stesso anno a Vienna, una terza forse in quegli stessi mesi a Zurigo; altre edizioni vennero stampate ad Ansbach nel 1788 e a Francoforte nel 1794. Si è a conoscenza di alcune occasionali recensioni e dei rapporti epistolari di Filangieri con intellettuali svizzeri e tedeschi, che però non sono sufficienti a chiarire ragioni e motivi di un così elevato numero di traduzioni e riedizioni nell'area tedesca (complessivamente almeno sette, fino alla metà dell'Ottocento). E certamente non bastano a spiegare un così precoce interesse per l'opera di Filangieri nell'area di lingua tedesca, se si tiene conto che, in proporzione, le versioni francesi e inglesi furono meno numerose. Alla fiera del libro di Francoforte e Lipsia della Pasqua 1784 la traduzione della "Scienza della legislazione" venne presentata come un evento editoriale, e il catalogo registrò tre concorrenti edizioni, quella viennese, quella svizzera e quella tedesca ${ }^{14}$.

Per quanto ne sappiamo, questi tre progetti editoriali furono non solo il risultato di una strategia interna al mercato librario, ma anche l'esito abbastanza diretto di un acceso dibattito, in corso in tutta l'area germanica, sull'idea della legge, sul significato dei processi di codificazione, sul mutamento dell'intero sistema costituzionale degli Stati di antico regime di fronte all'eversione del mondo feudale ${ }^{15}$. Si tratta di un dibattito già in gran parte conosciuto, segnato in Austria dalla riforma del processo civile sancita dalla "Civilgerichtsordnung" di Giuseppe II (1781), in Svizzera dal concorso sul progetto più esaustivo e dettagliato per una buona legislazione criminale indetto dalla società economica di Berna (1777$1778)^{16}$, in Prussia dall'avvio dei lavori per la redazione dell'Allgemeines Landrecht (1780).

${ }^{14}$ C. Filangieri, System der Gesetzgebung, aus dem Italienischen mit D. Siebenkees' Vorbericht. Anspach 1784, 2 voll.; Des Ritters Caietan Filangieri Wissenschaft der Gesetzgebung, aus dem Italienischen von Anton Gustermann, Wien 1784, un volume; C. Filangieri, Über die Gesetzgebung. Aus dem Italienischen. Zürich 1784. Per le stategie del mercato editoriale alle fiere del libro di Francoforte nel XVIII secolo cfr. D. Skala, Vom neuen Athen zur literarischen Provinz. Die Geschichte der Frankfurter Büchermesse bis ins 18. Jahrhundert, in: Brücke zwischen den Völkern. Zur Geschichte der Frankfurter Messe II, a cura di R. Косн. Frankfurt am Main 1991, 195-201.

${ }^{15}$ Per il dibattito relativo efr. P. Becchi-K. Seelmann, Gaetano Filangieri und die europäische Aufklärung (Rechtsphilosophische Schriften 8). Frankfurt am MainBerlin-Bern 2000; A. Trampus, La traduzione settecentesca di testi politici: il caso della "Scienza della legislazione". Rivista internazionale di tecnica della traduzione / International Journal of Translation 6 (2002), 19-44.

${ }^{16}$ Cfr. il Regolamento giudiziario di Giuseppe II (1781), rist. anastatica a cura di N. Pacardi (Testi e documenti per la storia del processo IV). Milano 1999. Sul concorso indetto dalla Società economica di Berna si vedano S. Schmid, Die 
Dopo esserci occupati in altra sede della traduzione in lingua tedesca apparsa ad Ansbach nel $1784^{17}$, che si affianca a quelle già da altri studiate per l'area spagnola, ci interesseremo in quest'occasione della fortuna dell'opera dell'illuminista napoletano nella monarchia asburgica e, più in particolare, della traduzione parziale pubblicata a Vienna nel 1784.

Come accennato, alla fiera del libro di Francoforte e Lipsia della Pasqua 1784 vennero presentate contemporaneamente ben tre edizioni, una viennese, una svizzera e una tedesca. Mentre la traduzione apparsa ad Ansbach è ben conosciuta ed oggetto di indagini, e quella svizzera sembra essere probabilmente soltanto un'edizione affidata per la vendita ai librai Gellner e Fuessli ma non da loro stampata, nulla si sapeva sinora di quella pubblicata a Vienna, che anzi tutti i commentatori davano per perduta o inesistente ${ }^{18}$. Essa non risulta presente nella Oesterreichische Nationalbibliothek né nelle biblioteche regionali austriache e neppure in quella del collegio Teresiano, l'istituzione presso la quale presumibilmente aveva lavorato il traduttore.

Il ritrovamento, avvenuto recentemente, di un esemplare del testo nella biblioteca comunale di Augsburg, seguito da quello di un secondo presso la Bayerische Staatsbibliothek, consente ora di restituire agli studi su Filangieri e sul contributo italiano all'Illuminismo europeo un capitolo finora sconosciuto e legato alla cultura austriaca.

L'opera reca il titolo "Die Wissenschaft / der / Gesezgebung / vom Ritter / Kajetan Filangieri. / Aus dem Italiänischen übersetzt / von / A. W. Gustermann". Il luogo di stampa è Vienna, in der Sonnleithnerischen Buchhandlung, con la data del 1784. Il frontespizio reca anche l'indicazione Erstes Buch, ed effettivamente si tratta della traduzione del primo libro, ma si tratta anche dell'unico effettivamente tradotto, perché gli altri non vennero portati a compimento.

Abhandlung von der Criminal-Gesetzgebung von Hanns von Globig und Johann Georg Huster. (Freiburger rechtsgeschichtliche Abhandlungen N.F. 14). Berlin 1990; W. Schild, 1577 Paragraphen aufgeklärter Strafrechtsvernunft. Zum ALR als philosophisches Strafgesetzbuch, in: Gemeinwohl, Freiheit, Vernunft, Rechtsstaat. 200 Jahre Allgemeines Landrecht für die Preussischen Staaten, a cura di F. Ebel. Berlin-New York 1995, 42, 52. Sulle vicende e sulle attività della società di Berna efr. E. Erne, Die schweizerischen Sozietäten. Lexikalische Darstellung der Reformgesellschaften des 18. Jahrhunderts in der Schweiz. Zürich 1988, $188-204$.

17 Trampus (come in nota 15), 19-44

${ }^{18}$ K. Seelmann, Gaetano Filangieri und die Proportionalität von Straftat und Strafe. Imputation und Prävention in der Strafrechtsphilosophie der Aufklärung, in: Gaetano Filangieri e l'Illuminismo europeo, a cura di L. D'Alessandro ( $\mathrm{La}$ boratorio 2). Napoli 1991, 529-566; Becchi-Seelmann (come in nota 15). 
Il volume risulta quindi tradotto da Anton Wilhelm Gustermann, un personaggio del quale non rimane alcuna traccia diretta nell'epistolario e nei documenti pervenutici di Gaetano Filangieri. Risulta quindi oltremodo difficile scoprire gli eventuali legami fra l'autore e il traduttore. Gustermann comunque non è un personaggio sconosciuto ai repertori biografici, nato a Vienna intorno al 1760 , aveva studiato nella capitale con la guida di Johann Heinrich van der Heyden, professore di diritto pubblico e feudale che poi lo aveva accolto come ripetitore per la medesima materia alla Wiener Hochschule. Nel 1796 avrebbe assunto un incarico di insegnamento al Theresianum e l'anno successivo un posto fisso in qualità di docente di diritto ecclesiastico, finché nel 1803 avrebbe accettato l'incarico di censore per gli studi giuridici e storici, mantenuto sino alla morte nel 1823. Fu autore di una „Kurze Geschichte Preussens mit Urkunden und Anmerkungen“ (Wien 1786) di un ,,Versuch eines vollständiges österreichischen Staatsrechts" (I. Theil, Wien 1793), dell" Oesterreichisches Kirchenrecht in den deutschen, ungarischen und galizischen Erbstaaten", (3 volumi, Wien 1812), dell'"Ausbildung der Verfassung des Königreichs Ungarn" (2 volumi, Wien 1811) e di varie altre opere giuridiche ${ }^{19}$.

Gustermann era quindi giurista all'inizio dell'attività, specializzato nel diritto pubblico e feudale, appena poco più giovane dell'altro coevo traduttore di Filangieri in lingua tedesca (autore della versione apparsa ad Anspach), cioè Gottlieb Christian Karl Link (1757-1798), nato ad Altdorf, studente dal 1776 nella locale università e rimasto, dopo la laurea nel 1778, sempre nella stessa cittadina come insegnante privato prima di trasferirsi a Norimberga, nel 1785, per esercitarvi la professione di avvocato. Alcune caratteristiche accomunavano dunque i primi traduttori di Filangieri a Vienna e a Ansbach: entrambi giovani, entrambi studiosi di diritto, tutti e due desiderosi di costruirsi una notorietà nei rispettivi paesi traducendo un'opera che stava suscitando discussioni, consensi e dissensi nel continente.

Il tipografo era Joseph Sonnleithner, attivo negli anni della cosiddetta "Pressefreiheit", fra il 1782 e il 1786, associato con Johann David Hörling nella gestione di una libreria a Vienna e noto anche come editore di alcuni

19 Formularbuch zu praktischen und gerichtlichen Aufsätzen in der k.k. Staaten, 2 voll. Wien 1812, Ungarisches Staatsrecht. Wien 1818; Oesterreichische Privatrechtpraxis, enthaltend das gerichtliche Verfahren in bürgerlichen Rechtssachen und den Justizgeschäftsstyl, 3 voll., ${ }^{2} 1805,{ }^{3} 1823$. Su di lui cfr. C. von WurzBAch, Biographisches Lexikon des Kaiserthums Oesterreichs VI. Wien 1860, 44-45; Oesterreichische National-Enzyklopedie von Gräffer und Czikann II. Wien 1835, 442. 
scritti di Joseph von Sonnenfels ${ }^{20}$. Proprio in chiusura della traduzione di questo volume della Scienza, alle pp. [1-2] vi è un catalogo dei libri recenti stampati a Vienna dalla libreria Sonnleithner e Hörling: "Neue Verlagartikel der Sonnleithner und Hörlingschen Buchhandlung in Wien", in cui compaiono oltre ad alcune traduzioni da Racine e da poeti inglesi, edizioni dei manuali di diritto di Karl Anton von Martini e un'opera storica di Joseph von Retzer, che riconducono tutte all'ambiente del collegio Teresiano.

L'analisi condotta in altra sede ha mostrato le ragioni che determinarono il successo della traduzione di Siebenkees e Link nel mondo tedesco, tanto da conoscere altre tre ristampe, nel 1788, 1794 e 1808: lo sforzo compiuto dai curatori per condurre il lavoro di traduzione non su un piano letterale ma su quello interpretativo, l'analisi del linguaggio di Filangieri e la ricerca della versione il più vicina possibile alla cultura tedesca. Un lavoro accompagnato da un'introduzione dei curatori (Siebenkees ne avrebbe aggiunta un'altra al secondo tomo), nella quale venivano sottolineati due aspetti della "Scienza della legislazione" che maggiormente potevano interessare il pubblico tedesco: il collegamento con la dottrina del diritto naturale e la dimensione storica del diritto, al centro degli interessi della scuola di Göttingen.

Diverso destino conobbe la traduzione austriaca di Gustermann: anzitutto non venne condotta a termine, e si arrestò al primo volume; in secondo luogo dovette avere una circolazione alquanto limitata, che potrebbe spiegare la rarità degli esemplari superstiti. Occorre allora cercare di capire le cause di questo insuccesso e di questa scarsa diffusione.

La breve presentazione di Gustermann premessa al volume denota il grado di conoscenza - a dire la verità piuttosto superficiale - dell'autore e della sua opera, anche se può sembrare un utile documento sulla raggiunta notorietà dell'illuminista napoletano. Dopo qualche informazione sulla fortuna editoriale di Filangieri nella penisola italiana (ma Gustermann sbagliava dicendo che il terzo volume della "Scienza" era stato pubblicato a Firenze nel 1783, città nella quale invece era apparsa una ristampa), affermava da subito quale era secondo lui il merito principale del lavoro: quello di affrontare e discutere criticamente l' "Esprit des lois", tanto da far valere all'autore l'appellativo di Montesquieu italiano. C'era una grande differenza tuttavia, osservava Gustermann, fra i due autori: riprendendo letteralmente il testo originale in italiano, spiegava che il

${ }^{20}$ N. Bachleitner, F. M. Eybl, E. Fischer, Geschichte des Buchhandels in Österreich. Wiesbaden 2000 (Geschichte des Buchhandels 6). 
filosofo francese si era limitato a mostrare quali erano le leggi e perché esse erano tali; mentre Filangieri aveva mostrato come dovevano essere e perché dovessero essere tali. Montesquieu aveva ragionato per idee e aforismi, Filangieri per princìpi giuridici e per verità.

Come aveva fatto in quegli stessi mesi anche il traduttore di Altdorf, Gustermann si preoccupava anche di illustrare e giustificare il suo lavoro di traduttore. Filangieri, affermava riprendendo ancora una volta il testo del napoletano, aveva parlato per l'umanità con toni appassionati. Egli, come traduttore, aveva fatto tutti gli sforzi possibili per trasferire questa passione sul piano linguistico, passo per passo ("Ich habe ... Period gegen Period übersezet"). Tuttavia aveva scelto di operare in modo diverso dal metodo traduttivo più in voga e utilizzato in particolare dai francesi, che si limitavano a parafrasare, cercando di restituire invece il significato autentico del testo filangieriano. Gustermann nulla diceva, invece, dell'edizione sulla quale aveva condotto la traduzione, anche se quell'unico accenno alla prima edizione napoletana - di cui si conservano peraltro alcuni esemplari ancora oggi nella Österreichische Nationalbibliothek, l'antica biblioteca di corte - lascia supporre che fosse proprio la prima ad essere utilizzata. Mancano ulteriori indicazioni sulle difficoltà e sulle scelte traduttive: il testo pubblicato a Vienna non presenta, a differenza della versione di Anspach, alcuna nota esplicativa o integrativa del traduttore; viceversa, talvolta Gustermann sembra aver preferito riportare direttamente nel testo, accanto a particolari concetti o parole da lui tradotti, anche l'originale italiano, così come appare sin dalla prima pagina ove, traducendo la frase "Si è proposta per oggetto di premio la scoverta d'una evoluzione più micidiale", propone "Man hat auf die Erfindung eines noch mörderischen Schlachten-Systems (evoluzione) einen Preis gesezet" 21 .

Un esame della traduzione e delle modalità con cui venne portata a compimento diventa decisivo allora per comprendere il livello del confronto tra il pensiero filangieriano e la cultura giuridica austriaca dell'epoca. È ciò che ci ripromettiamo di fare nelle pagine che seguono esaminando, relativamente al primo tomo dell'opera tradotta, il livello di intervento del traduttore attraverso le scelte e la resa lessicale di alcuni passaggi chiave.

Colpisce, anzitutto, la scelta di rendere il titolo dell'opera "Scienza della legislazione" con la traduzione "Wissenschaft der Gesezgebung", cioè "scienza", adottando un vero e proprio calco, anziché utilizzare

${ }^{21}$ Die Wissenschaft der Gesetzgebung (come in nota 14), 1. 
l'espressione System, "sistema" adottata ad Anspach dal traduttore tedesco Link, e che si ricollegava ad una diversa concezione del diritto. E non era un caso. Nella cultura austriaca il diritto veniva studiato preferibilmente nell'alveo delle scienze della politica secondo l'insegnamento di Joseph von Sonnenfels ${ }^{22}$; in quella tedesca emergeva l'aspirazione di ricondurre tutto il sapere giuridico ad un ordine sistematico, con maggiore attenzione per le implicazioni pratiche e legislative piuttosto che per quelle filosofico-giuridiche. A ben vedere, questa traduzione del titolo fatta da Gustermann è del tutto coerente con le scelte traduttive che emergono dal testo, ove il traduttore sembra aver preferito il significato politico dei termini rispetto a quello specificatamente giuridico. Così avviene per esempio all'inizio del capitolo terzo, dedicato alle regole generali della scienza legislativa e ai suoi difetti, dove Filangieri scrive: "L'indole dunque dell'uomo è incompatibile coll'esattezza e perfezione arbitraria. Ogni facoltà ha dovuto avere le sue regole ed a proporzione che si sono perfezionate le regole le facoltà si sono migliorate. La scienza della legislazione sarà forsi l'eccezione d'un principio così universale e costante?" (p. 73) 23 .

Qui l'autore spiega come, dovendosi considerare la legislazione una scienza al pari della geometria, dell'architettura, della pittura e della scultura, essa deve essere soggetta ad un insieme di regole, anziché all'arbitrio del legislatore. Gustermann traduce: "Die Neugung des Menschen leidet keine willkührliche Genauigkeit und Vollkommenheit. Jede Kunst muss ihre Regeln haben, und nach dem Verhältniss, als diese Regeln vollkommner wurden, nahmen auch die Künste an Vollkommenheit zu. Wäre vielleicht die Wissenschaft der Gesezgebung von diesem so allgemeinen und unveränderlichen Grundsaze ein Ausnahme?" (p. 90).

Il paragrafo mostra in maniera chiara, ci sembra, quale doveva essere stato l'impegno del traduttore per evitare calchi linguistici e per trasferire i significati da una lingua all'altra. Quella che per Filangieri era semplice "indole", cioè una naturale attitudine, diventava per il traduttore "Neugung" o per essere più precisi "Neigung", l'inclinazione dell'uomo. Appare evidente il tentativo di Gustermann di ricondurre il termine ad dibattito appena iniziato intorno alla filosofia kantiana (Schiller

${ }^{22}$ Cfr. M. R. Di Simone, Aspetti della cultura giuridica austriaca nel Settecento. Roma 1984

${ }^{23}$ G. Filangieri, La scienza della legislazione I. Napoli 1780. Nel prosieguo, i numeri di pagina delle citazioni in italiano si intendono riferiti a questa prima edizione italiana, quelli delle citazioni in lingua tedesca si intendono riferiti all'edizione di Vienna del 1784. 
riprenderà la riflessione su "Pflicht" e "Neigung" in "Über die ästhetische Erziehung des Menschen") di cui è difficile però capire quanto avesse consapevolezza. Anche nella traduzione del termine "facoltà" si avverte l'attenzione di Gustermann. Rendendosi conto che Filangieri intendeva riferirsi alle facoltà mentali dalle capacità raziocinanti dell'individuo, preferivano tradurre "facoltà" con "capacità" ("Kunst"), rispettando quindi l'opposizione tra "Kunst" e "Wissenschaft", laddove invece il traduttore tedesco nell'edizione di Anspach aveva usato qui e altrove sempre il termine "Wissenschaft".

Un'altra operazione interessante riguarda la traduzione di espressioni e di concetti che proprio in quell'epoca andavano caricandosi di nuove valenze: parole antiche, ma con significati nuovi. Può essere interessante verificare allora questo percorso esaminando tre concetti chiave del linguaggio politico illuminista, "lumi", "opinione pubblica" e "cittadino".

Filangieri, come noto, utilizza frequentemente la parola "lumi", un termine semanticamente ambiguo nella lingua italiana che infatti nella Scienza della legislazione è utilizzato sia come sinonimo di "idee", sia per indicare l'illuminazione dell'individuo per mezzo della fede e delle verità rivelate, sia infine per indicare l'illuminazione raggiungibile per mezzo della ragione ${ }^{24}$. Di tutto questo il curatore austriaco mostrava di avere consapevolezza, sapendo però di avere a disposizione anche un termine comparso più recentemente nella loro lingua come quello di "Aufklärung", dal significato assai più marcato rispetto all'equivalente italiano. Il dibattito berlinese sulla natura e sul significato dell'Aufklärung si era svolto, del resto, sulle pagine della rivista "Berlinische Monatsschrift" tra l'inverno del 1783 e la primavera del 1784, quindi proprio nel momento in cui veniva portata a termine la traduzione della "Scienza della legislazione". Oltre a ciò, Gustermann non poteva ignorare che proprio su una questione simile era intervenuto anche Aloys Blumauer, che solo due anni prima aveva pubblicato prima un saggio molto assai discusso intitolato "Beobachtungen über Österreichs Aufklärung und Litteratur"25.

${ }^{24}$ L’ingresso della "illuminismo" nella lingua italiana risale all'inizio del Novecento ed è collegata alle concettualizzazioni maturate all'interno della filosofia dello spirito di Benedetto Croce; ampi riferimenti sull'elaborazione di questa espressione nella cultura europea si possono trovare nella recente sintesi di V. Ferrone, D. Roche, L'Illuminismo nella cultura contemporanea. Storia e storiografia (Quadrante Laterza 116). Roma-Bari 2002.

${ }^{25}$ Sul testo e sul contesto culturale cfr. L. Bodi, Tauwetter in Wien. Zur Prosa der österreichischen Aufklärung 1781-1795 (Schriftenreihe der Österreichischen Gesellschaft zur Erforschung des 18. Jahrhunderts 6). Wien-Köln-Graz 1995; A. Trampus, I gesuiti e l'Illuminismo. Politica e religione in Austria e nell'Europa centrale 
Per questo, nella sua versione, Gustermann decise di optare per una evidente interpretazione prudente della parola lumi, ancora una volta differenziandosi dal traduttore di Anspach che invece aveva optato con maggiore decisione verso l'uso della parola "Aufklärung". Ecco un passo tratto dalle pagine iniziali: "Siccome lo Stato era allora diviso in tante porzioni per quanti feudi conteneva, siccome ciascheduna di queste parti era isolata, il talento, privo della comunicazione, si restringeva in una certa sfera di cognizioni e di lumi nella quale era costretto a fermarsi. La picciolezza medesima degl'interessi doveva allora indebolire gl'ingegni ed impedire che le idee si estendessero" (pp. 4-5).

Filangieri descriveva qui la condizione dell'individuo e della società nel mondo feudale, marcando la differenza rispetto al nascente mondo moderno. Ed ecco la traduzione: "Gleichwie der Staat damals in eben soviele Stücke getheilet war, als er Lehen enthielt, und jedes von der übrigen abgesondert war, eben so war der gute Kopf, weil er keine Gemeinschaft mit entfernten hatte, in einen kleinen Kreis der Kenntnisse eingeschränket, in dem er sich bilden musste. Selbst der geringe Vortheil, den damals die Kenntnisse verschaffen konnten, musste die Fähigkeit muthlos machen, und die Erweiterung der Begriffe hindern" (pp. 6-7).

L'espressione "lumi", contenuta nella prima parte della proposizione e riconducibile evidentemente ad un uso debole del termine, viene resa quindi con "Kenntnisse" (letteralmente "conoscenze"), mentre alla fine del brano la parola "idee", che il traduttore di Anspach più forzatamente traduceva come "Aufklärung", veniva resa con "Begriffe", cioè esattamente "idee" o "concetti". Lo stesso avviene anche più oltre quando, narrando dei progressi dell'economia europea e dei sistemi adottati per migliorarla, Filangieri afferma che il primo paese "a profittare di questi lumi fu l'Inghilterra" (p. 80), cioè "Engeland wusste zu erst daraus Vortheil zu ziehen" (p. 98).

Questo criterio trova conferma immediatamente dopo, ove il napoletano proseguiva nel suo ragionamento: "L'utilità pubblica richiedeva che si estirpasse tutto quello che si opponeva a' progressi de' lumi e delle cognizioni, senza de' quali ogni riforma, e particolarmente quella delle leggi, sarebbe stata difettosa e funesta" (p. 6).

Filangieri elencava dunque gli ostacoli che nel passato si erano frapposti alla riforma della legislazione, indicando i mezzi per superarli. Il traduttore austriaco volgeva: "Das allgemeine Wohl forderte alles dasjenige aus dem Wege zu räumen, was der Verbreitung der Aufklärung

1773-1798 (Università degli Studi di Torino, Fondo di studi Parini-Chirio, Storia 5). Firenze 2000, 217-262. 
und der Kenntnisse entgegenstund, ohne welche jede Verbesserung, besonders in den Gesezen mangelhaft, und schädlich gewesen sein wurde" (p. 8).

In questo caso Gustermann si rivela attento nel rimanere aderente alla lettera del testo filangieriano e ad attribuire alla parola "lumi" il suo vero significato. Tale procedimento, basato su una preventiva analisi del significato dell'espressione "lumi" e sulla scelta, per la traduzione, di un termine semanticamente "forte" come "Aufklärung", viene applicato anche alla fine del capo quarto: "i progressi de" lumi e della filosofia, uniti alle virtù de' troni, ci fanno sperare che il suo esempio sarà imittato dal resto delle nazioni" (p. 100).

Filangieri si riferiva qui ai diritti di libertà garantiti dalla costituzione della Pennsylvania, esempio per la legislazione dei paesi europei. Nella versione austriaca ciò diventa: "Der Fortgang der Aufklärung, die Verbreitung der Philosophie, und tugendhafte Fürsten auf dem Throne lassen uns hoffen, dass auch die übrigen Völker dem Beispiele der Pensilvanier folgen werden" (p. 123).

Un altro importante esempio delle scelte traduttive riguarda il tema dell'opinione pubblica. Come è noto, nel volgere del Settecento e a partire soprattutto dall'area inglese, si assistette alla lenta trasformazione del concetto di opinione generale o pubblica, intesa in senso prevalentemente negativo come opposta alla verità, ad opinione pubblica critica con significato politico ${ }^{26}$. Questo mutamento di significato si registra sia a livello politico sia sul piano giuridico, con ripercussioni sulla configurazione del delitto d'infamia, che veniva ridefinito - superando l'antico confronto tra infamia juris e infamia facti - come delitto d'opinione, qualificandolo giuridicamente attraverso il criterio della reputazione. Nello spazio italiano è proprio Filangieri a far intravedere questa trasformazione di significati, pur tra qualche contraddizione. Il traduttore, nel rendere il linguaggio filangieriano, avvertiva le oscillazioni lessicali e cercava di adottare una precisa gradazione nell'uso delle parole; come avviene nel capitolo VII, relativo ai mezzi per superare gli ostacoli che si oppongono al cambiamento della legislazione: "Se la legislazione opera allorché persuade; se i voti del pubblico non sono indifferenti per le leggi ... “ (p. 111).

${ }^{26}$ E. Tortarolo, Censorship and the Conception of the Public in the Late Eighteenth-Century Germany: Or, are Censorship and Public Opinion Mutually Exclusive?, in: Shifting the Boundaries. Transformation of the Languages of Public and Private in the Eighteenth Century, a cura di D. Castiglione-L. Sharpe. Exeter 1996, 131-150. 
Il passo si riferisce alla necessità di creare il consenso nel momento in cui si procede ad una riforma della legislazione e il traduttore lo volge: "Wenn es wahr ist, dass die Gesezgebung damals am beste wirkte, wenn sie überredet, und schon selbst die Wünsche der Nazion gewissen Gesezen entgegen sehen ..." (p. 136).

Il pubblico cui fa riferimento qui Filangieri non è ancora l'opinione pubblica, depositaria o espressione della volontà popolare; per lui era più semplicemente l'opinione generale. Nella traduzione, il "pubblico" non tendenzialmente critico, che cioè non si assume il controllo politico degli atti di governo, diventa per Gustermann come si è visto "die Nazion". Poco più avanti però, nello stesso capitolo, "se agire senza consultare la volontà de' popoli e senza raccogliere per così dire la pluralità de' suffragi dell'opinione pubblica è un errore ... " (pp. 111-112).

In questo caso, come si vede, l'opinione pubblica diventa uno strumento di equilibrio fra i poteri e di controllo politico degli atti legislativi; ma la traduzione non cambia: "Wenn alle Neuerungen, wären sie auch noch so nothwendig, missfallen, wenn sie die Nazion nicht gleichsam laut forderet, oder wenn sie nicht wenigstens allgemein gewünscht werden ..." (p. 136-137).

La scarsa caratterizzazione concettuale tra un pubblico non necessariamente critico, ma piuttosto condizionabile dal messaggio politico, rispetto ad un pubblico capace di manifestare un suo consenso verso l'azione di governo emerge ancora più chiaramente poco più avanti: "Non basta persuadere il pubblico contro l'antica legislazione, bisogna prevenirlo in favore della nuova. Gli argomenti per ottenere questa necessaria prevenzione debbono essere sensibili. Essi debbono nascere dall'opinione istessa" (p. 114).

Gustermann, dovendo tradurre questa coppia di sostantivi marcandone il diverso significato, mantiene l'opposizione utilizzando due espressioni diverse, ma ancora una volta senza ricorrere mai a quella di "opinione pubblica": "Es ist nicht genug das Publikum wider die alte Gesezgebung einnehmen, man muss es auch für die Neuen vorhinein zu gewinnen suchen. Die Gründe dieses zu erreichen, müssen aber einleuchtend sein. Sie müssen aus dem Wahne der Nazion selbst hergenommen werden" (pp. 140-151).

In nessuno dei casi ora esaminati, Gustermann traduce quindi "pubblico" o "pubblica opinione" con "öffentliche Meinung": questo rappresenta una notevole differenza rispetto alla scelta del traduttore tedesco di Ansbach che già nel primo, ma poi soprattutto nel terzo libro della "Scienza della legislazione", soprattutto nel capitolo XXXI relativo alle pene d'infamia e alla perdita delle libertà civili come conseguenza di un 
delitto, aveva operato diversamente e con maggiore decisione. Qui il traduttore Link avrebbe confermato tutte le scelte lessicali già adottate nel libro I, alternando fra loro le espressioni "Opinion"/"opinione", "Opinion des Publikums"/"opinione del pubblico", e "öffentliche Meinung"/"opinione pubblica".

Se ne trae l'impressione, quindi, che al momento di proporre il testo di Filangieri in Austria, Gustermann era forse ancora poco informato sui dibattiti concettuali e lessicali degli ambienti illuministi berlinesi, oppure intenzionalmente avesse mantenuto un profilo più basso nella scelta dei termini utilizzati per rendere il linguaggio filangieriano.

Il terzo aspetto su cui può essere interessante soffermarsi riguarda il nuovo significato di un'altra parola di antico uso, cioè l'espressione "cittadinanza". Come noto, nel linguaggio politico settecentesco all'originaria distinzione tra "suddito" e "cittadino" seguì ben presto un'ulteriore differenziazione nel significato del termine "cittadino". Da una parte, conformemente all'uso più antico, continuò a mantenere il senso di titolare dei diritti di cittadinanza (nelle città, nei comuni); dall'altra, cominciava ad indicare anche chi fruiva, all'interno della compagine dello Stato, delle libertà del cittadino pur senza ancora partecipare direttamente alle decisioni politiche. Rousseau, nel "Contrat social", aveva sottolineato già il fatto che la moderna idea di cittadinanza e il concetto di cittadino presupponevano inscindibilmente le libertà politiche e i diritti derivanti dall'essere membri del corpo politico e dalla partecipazione alla sovranità. Tuttavia, nel contesto della cultura dell'assolutismo italiano e prussiano, la trasposizione di questi concetti non appariva affatto facile, anche a causa di una più lenta elaborazione del linguaggio politico; non è dunque un caso che Filangieri nella sua opera usasse spesso il termine "cittadino" senza sfumarne eccessivamente il significato.

Nel mondo germanico, la polarizzazione tra l'idea del "cittadino" titolare dei diritti di cittadinanza e del "cittadino" dotato di diritti politici e partecipe della sovranità divenne evidente - come già altri hanno notato - soltanto negli anni Sessanta del Settecento e soprattutto nell'ambito della discussione antirousseauiana. In tale contesto, autori come Heinrich Gottfried Scheidemantel ("Das Staatsrecht nach der Vernunft und den Sitten der vornehmsten Völker" betrachtet, 1770-1773) e Johann Heinrich Gottlob Justi ("Natur und Wesen des Staates", 1771) cominciarono a introdurre la distinzione tra "Bürger", cioè titolare dei diritti storici di cittadinanza (corrispondente al francese "bourgeois"), e "Staatsbürger" (cittadino dello Stato), corrispettivo del francese "citoyen". Il significato risultava però almeno in parte attenuato rispetto a quello dell'espressione francese: "Staatsbürger", infatti, non era necessa- 
riamente chi partecipava al governo politico del paese ma, più in generale, chi svolgeva un qualsiasi ruolo giuridico e politico, mobilitandosi per esempio attraverso l'opinione pubblica. In questo modo, nella lingua tedesca, il termine "cittadino" ("Staatsbürger") veniva "intenzionalmente collocato in una posizione mediana tra "suddito" e "citoyen" 27.

Nella traduzione in lingua tedesca del testo di Filangieri questo livello della discussione è percepibile solo in parte. Il traduttore di Ansbach, Link, aveva utilizzato esclusivamente l'espressione "Bürger", tanto per indicare il cittadino in senso rousseauiano ("bourgeois"), quanto per indicare l'abitante delle città-Stato antiche. Così era avvenuto, per esempio, nel capitolo II di "System der Gesezgebung", dove si studia la ricchezza come mezzo per assicurare la tranquillità e la conservazione dei cittadini (p. 85). Qualche volta, però, il traduttore riusciva a connotare in modo più forte l'espressione "Bürger" o attraverso l'aggettivazione, o attraverso un complemento di specificazione. Si veda ad esempio nel capitolo VIII, dove Filangieri proponeva un sistema di verifica della legittimità costituzionale delle norme attraverso l'istituzione di un "censore delle leggi": "Questa magistratura, composta da' più savi e più illuminati cittadini dello Stato, potrebbe avere la maggiore influenza su la perpetuità dell'ordine legale" (p. 122).

Qui l'espressione "cittadino" non aveva un senso storico, ma serviva a distinguere l'individuo che, attraverso la propria appartenenza a questa nuova magistratura, poteva partecipare effettivamente della sovranità. Non a caso Filangieri stesso specificava che si trattava di "cittadini dello Stato", cioè proprio di un corrispondente del "citoyen" francese. Per il traduttore austriaco era quindi sufficiente proporre un calco e volgerlo con un "Bürger des Staats": "Diese müsste ein Magistrat haben, der aus den weisesten und aufgeklärtesten Bürgern des Staates bestünde, und dieser könnte auf die Dauer der gesezmässigen Ordnung den stärksten Einfluß haben" (p. 150).

Una conferma di questa scelta si ritrova nel capitolo XI, dove Filangieri criticava la costituzione inglese e in particolare la complicità di Enrico VIII con il parlamento a danno delle libertà del popolo: "Non fu forse colle mani delle due camere che egli accese i roghi, dove i migliori cittadini dello Stato andavano a terminare i loro giorni?" (pp. 161-162).

${ }^{27}$ M. Stolleis, Stato e ragion di Stato nella prima età moderna (Collezione di testi e sturi. Filosofia). Bologna 1998, 297-338; IdEM, Konstitution und Intervention. Studien zur Geschichte des öffentlichen Rechts im 19. Jahrhundert (Suhrkamp Taschenbuch Wissenschaft 1526). Frankfurt am Main 2001, 33-46. 
Quindi: “Zündete er nicht mit den Händen dieser zwei Häuser die Scheiterhauffen an, auf denen die vorttrefflichsten Bürger ihr Leben liessen?" (p. 199).

Esaminati questi aspetti, che ci mostrano in quale misura le scelte traduttive della "Scienza della legislazione" in Austria avessero recepito le trasformazioni del linguaggio politico europeo ma anche del linguaggio giuridico tedesco, possiamo vedere più direttamente altri aspetti della traduzione, che si riferiscono piuttosto al dibattito in corso nell'area prussiana sulla nuova politica costituzionale degli anni Ottanta. Si tratta di un'indagine che può essere agevolmente condotta, sempre sulla scorta del libro I della "Scienza", esaminando il lessico relativo all'ordinamento e all'organizzazione dello Stato, ed espressioni quali "amministrazione", "governo", "legge" e "costituzione" lo dimostrano.

Riprendiamo il testo di Filangieri dal suo inizio, in particolare dove traccia il piano generale dell'opera e annuncia il contenuto del tomo II: "Quali sono gli ostacoli che si oppongono dagli abusi dell'amministrazione, dalla soverchia ingerenza del governo, dalla stranezza delle leggi civili, dalla barbarie de' codici feudali ...?" (p. 30).

L'autore comincia ad elencare, in questo passo, quelli che dovrebbero essere i primi compiti del nuovo legislatore che si appresta a riformare lo Stato. Nella traduzione di Gustermann ciò diventa: "Was für Hindernisse zeigen sich dagegen von Seite der Misbräuche in der Verwaltung, von der Regirung, die sich ohne Nothwendigkeit darein menget, von der Unschicklichkeit der bürgerlichen und Lehengeseze ...?" (p. 37).

Abbiamo sottolineato questo paragrafo perché rivela molto chiaramente la scelta lessicale adottata da Gustermann: traduceva "amministrazione" con "Verwaltung" (amministrazione statale), adoperando un termine che in altre parti del testo, normalmente, utilizzava per rendere piuttosto l'espressione "governo". E rendeva "governo" con "Regirung", traducendo quasi letteralmente il concetto filangieriano di "stranezza delle leggi civili'e dei codici feudali ("Unschicklichkeit der bürgerlichen und Lehengeseze") laddove invece il traduttore di Ansbach aveva fatto una vera e propria interpretazione del testo mutandone il significato.

Questa aderenza al significato letterale delle parole consentiva a Gustermann di evitare quindi sia note esplicative, sia interventi o cesure al testo originale. Ad esempio, Filangieri aveva scritto: "Siccome l'esame del rapporto delle leggi colla natura d'un governo non è altro, che l'esame de’ principi e delle regole, che fan conoscere al legislatore i difetti della sua costituzione e i rimedi propri per correggerli ..." (p. 152).

La traduzione di Ansbach, nello sforzo di adattare la frase ad un contesto maggiormente specificato sul piano linguistico aveva portato a 
far cadere una parte dell'oggetto, quella riguardante i "difetti della costituzione". Gustermann invece scriveva: "Das Verhältniss der Geseze mit der Natur einer Regirungsart untersuchen, heisst die Grundsäze und Regeln aufsuchen, welche dem Gesezgeber die Gebrechen seiner Staatsverfassung, und die tauglichsten Mittel, selbe zu verbessern, anzeigen" (p. 187).

In questo modo il verbo "verbessern" mantiene il referente originale: sono i difetti della costituzione a dover essere corretti e non i principi e le regole che stanno alla base del governo.

Il problema della traduzione della parola "costituzione" ci porta, attraverso il confronto tra i due testi, a comprendere anche in quale misura la versione austriaca non soltanto volgeva il testo filangieriano, ma serviva a registrare anche la nascita del nuovo linguaggio costituzionale degli anni Ottanta.

Nella lingua tedesca si assiste infatti proprio in quegli anni alla graduale comparsa del termine "Konstitution" in senso politico e giuridico. Sino alla fine del XVIII secolo la parola "Konstitution" era stata utilizzata quasi esclusivamente nel senso di "costituzione fisica dell'individuo" ("Leibeskonstitution"). Quando si voleva fare riferimento ad atti legislativi, si preferiva fare ricorso al latino "constitutio", e l'uso di questa parola rimaneva limitato esclusivamente all'ambito della legge imperiale ("Kaisergesetz"), contrapposta alle leggi statutarie e al diritto comune ${ }^{28}$. Nelle lingue neolatine invece, e particolarmente in quella francese, l'uso del termine "costituzione" nel senso di "legge fondamentale dello Stato" sembra fosse entrato trent'anni prima attraverso l'opera di Emer de Vattel, Les doit des gens ou principes de la loi naturelle del $1758^{29}$, ma nello spazio tedesco la parola "Konstitution" cominciò ad acquistare il significato di "legge fondamentale dello Stato" ("Staatsgrundgesetz") molto più tardi, per trovare accoglienza definitiva appena negli anni Novanta e prevalentemente nell'ambito del pensiero repubblicano, con occasionali citazioni in Häberlin, Wieland (1793) e, dal 1795, in Kant ${ }^{30}$, per essere poi consacrato dal "Wilhelm Meister" di Goethe del 1796.

\footnotetext{
28 A. Erler, Konstitution, Constitutio, voce in: Handwörterbuch zur Deutschen Rechtsgeschichte V, a cura di A. Erler-E. Kaufmann-D. Werkmüller. Berlin 1978, 1119-1122; Etymologisches Wörterbuch des Deutschen, a cura di W. PfeIFER. Berlin 1993, 710.

${ }^{29}$ H. Монnhaupt, Verfassung (I.), in: Geschichtliche Grundbegriffe, Bd. VI, a cura di W. Conze-R. Koseldeck. Stuttgart 1990, 858-870.

${ }^{30}$ Kant utilizza il termine "Konstitution" come sinonimo di "Verfassung" nel suo progetto di pace perpetua, là dove afferma il valore della costituzione repubblicana, espressione della sovranità popolare e garanzia delle libertà dell'uomo.
} 
Secondo le fonti note, la prima testimonianza assoluta del nuovo uso della parola costituzione sarebbe costituita proprio da un saggio di Johann Georg Schlosser contro Filangieri e contro la codificazione prussiana, apparso nel $1789^{31}$. Allora, senza voler enfatizzare eccessivamente la primogenitura di questa citazione, si può certamente ipotizzare che un contributo all'elaborazione del linguaggio politico costituzionale nello spazio tedesco possa essere giunto proprio dalle traduzioni della "Scienza della legislazione". Nella traduzione tedesca pubblicata ad Ansbach nel 1784, ad esempio, si può ben notare come avesse cominciato già ad utilizzare - siamo nel 1784 - accanto a "Verfassung" (la costituzione in senso materiale, cioè l'ordinamento dello Stato), anche "Konstitution", nel senso moderno di costituzione quale legge fondamentale del paese.

Vediamo alcuni esempi tratti dalla traduzione austriaca della "Scienza". Filangieri utilizzava la stessa parola sia per indicare la costituzione materiale, non scritta, quindi la natura dei governi, come faceva quando scriveva della molteplicità e della diversità delle costituzioni (p. 151), sia per individuare le regole fondamentali dello Stato, là dove sosteneva che la legislazione non deve mutare l'essenza della costituzione, deve solo correggerne i difetti (p. 154). Il traduttore austriaco cercava di rendere il differente uso di questa parola optando per soluzioni lessicali diverse. Nel primo caso traduceva "costituzione" con "Regirungsarten", cioè modo di governare (p. 186), mentre nel secondo caso preferiva l'espressione "Verfassung", cioè - correttamente - costituzione (p. 190).

Gustermann optava quindi per un tipo di traduzione che potesse rendere il testo di Filangieri comprensibile al pubblico austriaco. Infatti l'espressione "Verfassung", come veniva adoperata in questo caso, richiamava un linguaggio giuridico già in uso, in particolare quello che si riferiva al sistema del diritto pubblico dell'impero, cioè alle norme scaturite dalla pace di Westfalia sulle quali si reggevano la complessa architettura dell'impero e la "Reichsverfassung", cioè il suo assetto costituzionale ${ }^{32}$.

31 J. G. Schlosser, Briefe über die Gesetzgebung überhaupt und den Entwurf des preussischen Gesetzbuchs insbesondere. Frankfurt 1789, 1-81. In questo senso la si ritrova frequentemente della pubblicistica dell'epoca. Cfr. ad esempio Über die innere Verfassung der Preussischen Staaten. Der deutsche Zuschauer 1 (1785), 164 ss.; Wenn hat eine Nation das Recht, sich eine neue Konstitution zu geben. Neues Staatenjournal 10 (1789), 10-15.

${ }^{32}$ M. Stolleis, Geschichte des öffentlichen Rechts in Deutschland I, Reichspublizistik und Policeywissenschaft 1600-1800. München 1988, 155-156, che analizza anche il linguaggio giuridico dell'Impero. 
Mentre nella traduzione di Anspach compariva quindi già il termine "Konstitution" per indicare la costituzione scritta così come la intendeva Filangieri, nella traduzione viennese Gustermann continua a utilizzare solo ed esclusivamente la parola "Verfassung", riferita sia all'idea della costituzione materiale, sia a quella di legge fondamentale dello Stato. Perciò, nel punto in cui Filangieri aveva commentato la costituzione inglese e gli attentati politici mossi contro questa da Giacomo II (p. 157), Gustermann per non ripetere la stessa parola era costretto ad usare un un termine equivalente ma non sinonimo: "L'incostanza dunque della costituzione è il terzo vizio inerente alla costituzione di questa specie di governo che la legislazione deve riparare" (p. 167).

E allora il traduttore austriaco ricorreva alla parola "Regirungsart" per indicare la costituzione materiale o forma di governo da migliorare: "Diese Unsicherheit der Reichsverfassung ist also das dritte Gebrechen der vermischten Regirungsarten, welchem die Gesezgebung abhelffen muß" (p. 205).

Come si vede, pur riferendosi ad una realtà specifica come quella inglese, Gustermann utilizza un termine quale "Reichsverfassung" che si riferisce squisitamente all'area germanica. Si tratta di un passaggio chiave, perché corrisponde al punto in cui Filangieri, dopo aver esaminato e discusso i pregi e i difetti della costituzione inglese, estendeva il ragionamento ai sistemi politici del continente europeo, per esaminarne i principi costituzionali e auspicare la formazione di "un piccolo codice a parte delle vere leggi fondamentali che determinassero la vera natura della costituzione" (p. 103) che nella traduzione di Gustermann diventa "eine kleine Sammlung der wahren Fundamentalgeseze machen, nach welcher sich die wahre Staatsverfassung ... bestimmen liessen" (p. 220).

Sarebbe interessante poter approfondire le motivazioni di carattere culturale e giuridico che potevano aver spinto Gustermann a scegliere il termine "Reichsverfassung" e a respingere, eventualmente, quello di "Konstitution" già in uso altrove nell'area germanica, anche da parte di altri traduttori di Filangieri. Molto probabilmente vi si può riconoscere l'influenza dell'insegnamento del diritto pubblico nelle scuole e nelle università asburgiche. Certo è, ad ogni modo, che la traduzione viennese del primo volume dell'opera di Filangieri restituisce una dimensione culturale nella quale ancora faticava ad affermarsi il linguaggio moderno dell'Illuminismo e che costringeva il traduttore a depotenziare talvolta il significato delle espressioni filangieriane. Una difficoltà con la quale, viceversa, non dovevano fare i conti i traduttori di Ansbach, la cui versione in lingua tedesca, forse più coraggiosa sul piano delle scelte linguistiche, avrebbe conosciuto maggior fortuna e diffusione. 